\title{
PARTIAL SUMS FOR CERTAIN CLASSES OF MEROMORPHIC FUNCTIONS
}

\author{
RABHA W. IBRAHIM AND MASLINA DARUS
}

\begin{abstract}
In this paper, we define and study new classes of meromorphic functions in the punctured disk by using their partial sums.
\end{abstract}

\section{Introduction}

Let $\Sigma_{\alpha}$ denote the class of functions of the form

$$
f(z)=\frac{1}{z^{1+\alpha}}+\sum_{n=1}^{\infty} a_{n} z^{n+\alpha}, \quad(0 \leq \alpha<1,),
$$

which are analytic in the punctured unit disk $U:=\{z \in \mathbb{C}, 0<|z|<1\}$.

A function $f \in \Sigma_{\alpha}$ belongs to the class $\mathcal{S}_{\alpha}(A, B)$, the class of meromorphically $\alpha$ valent starlike functions if and only if $f \neq 0$, and for $-1 \leq A<B \leq 1$,

$$
-\left\{\frac{z f^{\prime}(z)}{f(z)}\right\} \prec \frac{1+A z}{1+B z}, \quad(z \in U) .
$$

A function $f \in \Sigma_{\alpha}$ belongs to the class $\mathcal{C}_{\alpha}(A, B)$, the class of meromorphically $\alpha$-valent convex functions if and only if $f^{\prime} \neq 0$, and

$$
-\left\{1+\frac{z f^{\prime \prime}(z)}{f^{\prime}(z)}\right\} \prec \frac{1+A z}{1+B z}, \quad(z \in U) .
$$

The class $\Sigma_{0} \equiv \Sigma$, was studied by many authors (see $[1,2,3,4,5]$ ). Note that the authors defined and studied the class $\Sigma_{\alpha}$ for normalized analytic functions in an open disk (see $[6,7])$.

In the present paper, we are motivated with the work done by Silverman [8], and will investigate in similar manner the ratio of a function of the form (1) to its sequence of partial sums

$$
f_{k}(z)=\frac{1}{z^{1+\alpha}}+\sum_{n=1}^{k} a_{n} z^{n+\alpha}, \quad(0 \leq \alpha<1,),
$$

Corresponding author: Maslina Darus.

Received February 17, 2008; revised June 13, 2009.

2000 Mathematics Subject Classification. 30C45.

Key words and phrases. Partial sum, meromorphic functions. 
when the coefficients are sufficiently small. More precisely, we will determine sharp lower bounds for

$$
\Re\left\{\frac{f(z)}{f_{k}(z)}\right\}, \Re\left\{\frac{f_{k}(z)}{f(z)}\right\}, \Re\left\{\frac{f^{\prime}(z)}{f_{k}^{\prime}(z)}\right\} \text {, and } \Re\left\{\frac{f_{k}^{\prime}(z)}{f^{\prime}(z)}\right\} .
$$

\section{Preliminary results}

First we prove sufficient conditions for $f \in \Sigma_{\alpha}$ to be in the classes $\mathcal{S}_{\alpha}(A, B)$ and $\mathcal{C}_{\alpha}(A, B)$

Theorem 2.1. Let $f \in \Sigma_{\alpha}$. If

$$
\sum_{n=1}^{\infty}[(n+\alpha)(1+B)+(A+1)]\left|a_{n}\right| \leq(B-A)-\alpha(1-B), \quad(z \in U)
$$

holds and $B(1+\alpha)>A+\alpha$, then $f \in \mathcal{S}_{\alpha}(A, B)$.

Proof. Assume that $f \in \Sigma_{\alpha}$ and satisfies (3). It is sufficient to show that

$$
\left|\frac{1+\frac{z f^{\prime}(z)}{f(z)}}{B\left(\frac{z f^{\prime}(z)}{f(z)}\right)+A}\right|<1
$$

that is

$$
\left|\frac{f(z)+z f^{\prime}(z)}{A f(z)+B z f^{\prime}(z)}\right|<1
$$

Consider

$$
\begin{aligned}
\left|\frac{f(z)+z f^{\prime}(z)}{A f(z)+B z f^{\prime}(z)}\right| & =\left|\frac{-\frac{\alpha}{z^{\alpha+1}}+\sum_{n=1}^{\infty} a_{n}(n+\alpha+1) z^{n+\alpha}}{\frac{A}{z^{\alpha+1}}+\sum_{n=1}^{\infty} A a_{n} z^{n+\alpha}-\frac{B(1+\alpha)}{z^{\alpha+1}}+\sum_{n=1}^{\infty}(n+\alpha) B a_{n} z^{n+\alpha}}\right| \\
& \leq \frac{\alpha+\sum_{n=1}^{\infty}(n+\alpha+1)\left|a_{n}\right|}{[B(1+\alpha)-A]-\sum_{n=1}^{\infty}[(n+\alpha) B+A]\left|a_{n}\right|} .
\end{aligned}
$$

Hence (4) is bounded by 1 , if

$$
\begin{aligned}
& \alpha+\sum_{n=1}^{\infty}(n+\alpha+1)\left|a_{n}\right| \leq[B(1+\alpha)-A]-\sum_{n=1}^{\infty}[(n+\alpha) B+A]\left|a_{n}\right| \\
& \Rightarrow \sum_{n=1}^{\infty}(n+\alpha+1)\left|a_{n}\right|+\sum_{n=1}^{\infty}[(n+\alpha) B+A]\left|a_{n}\right| \leq[B(1+\alpha)-A]-\alpha \\
& \Rightarrow \sum_{n=1}^{\infty}[(n+\alpha)(1+B)+(1+A)]\left|a_{n}\right| \leq(B-A)-\alpha(1-B),
\end{aligned}
$$

where $B(1+\alpha)>A+\alpha$. This completes the proof.

In a similar manner, we can prove the following result 
Theorem 2.2. Let $f \in \Sigma_{\alpha}$. If

$$
\sum_{n=1}^{\infty}(n+\alpha)[(n+\alpha)(1+B)+(A+1)]\left|a_{n}\right| \leq(1+\alpha)[(B-A)-\alpha(1-B)], \quad(z \in U)
$$

holds and $B(1+\alpha)>A+\alpha$, then $f \in \mathcal{C}_{\alpha}(A, B)$.

Note that when $\alpha=0$, Theorem 2.1 and Theorem 2.2 reduce to Theorem 2.2 and Theorem 2.1 in [5] respectively. Further, we note that these sufficient conditions are also necessary for functions of the form (1) when $\alpha=0, A=2 \mu-1, B=1$ with positive or negative coefficients $([1,2,3])$.

\section{Main results}

We consider in this section partial sums of functions in the classes $\mathcal{S}_{\alpha}(A, B)$ and $\mathcal{C}_{\alpha}(A, B)$ and obtain the sharp lower bounds for the ratio of real part of $f(z)$ to $f_{k}(z)$ and $f^{\prime}(z)$ to $f_{k}^{\prime}(z)$. In the sequel, we will make use of the generalized result such that $\Re\left\{\left(1+w_{\alpha}(z)\right) /\left(1-w_{\alpha}(z)\right)\right\}>0, \quad(z \in U)$ if and only if $w_{\alpha}(z)=\sum_{n=1}^{\infty} c_{n} z^{n+\alpha}$ satisfies the inequality $\left|w_{\alpha}(z)\right|<|z|$.

Theorem 3.1. Let $f$ be given by (1) and satisfies (3) then

$$
\Re\left\{\frac{f(z)}{f_{k}(z)}\right\} \geq \frac{2(1+k+\alpha+A)}{2(k+\alpha)+(2+A+B)}, \quad(z \in U) .
$$

The result is sharp for every $k$ with extremal function

$$
f(z)=\frac{1}{z^{1+\alpha}}+\frac{(B-A)-\alpha(1-B)}{2(k+\alpha)+(2+A+B)} z^{k+1+\alpha}, \quad k \geq 0 .
$$

Proof. Assume that $f \in \Sigma_{\alpha}$ and satisfies (3). Consider

$$
\begin{aligned}
\frac{2(k+\alpha)+(2+A+B)}{(B-A)-\alpha(1-B)} & {\left[\frac{f(z)}{f_{k}(z)}-\frac{2(1+k+\alpha+A)}{2(k+\alpha)+(2+A+B)}\right] } \\
& =\frac{1+\sum_{n=1}^{k} a_{n} z^{n+2 \alpha+1}+\frac{2(k+\alpha)+(2+A+B)}{(B-A)-\alpha(1-B)} \sum_{n=k+1}^{\infty} a_{n} z^{n+2 \alpha+1}}{1+\sum_{n=1}^{k} a_{n} z^{n+2 \alpha+1}} \\
& :=\frac{1+w_{\alpha}(z)}{1-w_{\alpha}(z)}
\end{aligned}
$$

where

$$
w_{\alpha}(z)=\frac{\frac{2(k+\alpha)+(2+A+B)}{(B-A)-\alpha(1-B)} \sum_{n=k+1}^{\infty} a_{n} z^{n+2 \alpha+1}}{2+2 \sum_{n=1}^{k} a_{n} z^{n+2 \alpha+1}+\frac{2(k+\alpha)+(2+A+B)}{(B-A)-\alpha(1-B)} \sum_{n=k+1}^{\infty} a_{n} z^{n+2 \alpha+1}}
$$


and

$$
\left|w_{\alpha}(z)\right| \leq \frac{\frac{2(k+\alpha)+(2+A+B)}{(B-A)-\alpha(1-B)} \sum_{n=k+1}^{\infty}\left|a_{n}\right|}{2-2 \sum_{n=1}^{k}\left|a_{n}\right|-\frac{2(k+\alpha)+(2+A+B)}{(B-A)-\alpha(1-B)} \sum_{n=k+1}^{\infty}\left|a_{n}\right|} .
$$

Now $\left|w_{\alpha}(z)\right| \leq 1$ if and only if

$$
2\left[\frac{2(k+\alpha)+(2+A+B)}{(B-A)-\alpha(1-B)}\right] \sum_{n=k+1}^{\infty}\left|a_{n}\right| \leq 2-2 \sum_{n=1}^{k}\left|a_{n}\right|
$$

which is equivalent to

$$
\sum_{n=1}^{k}\left|a_{n}\right|+\left[\frac{2(k+\alpha)+(2+A+B)}{(B-A)-\alpha(1-B)}\right] \sum_{n=k+1}^{\infty}\left|a_{n}\right| \leq 1 .
$$

It is suffices to show that the left hand side of (8) is bounded above by

$$
\sum_{n=1}^{\infty}\left[\frac{2(n+\alpha)+A+B}{(B-A)-\alpha(1-B)}\right]\left|a_{n}\right|
$$

which is equivalent

$$
\sum_{n=1}^{k}\left[\frac{2(n+A+\alpha)}{(B-A)-\alpha(1-B)}\right]\left|a_{n}\right|+\sum_{n=k+1}^{\infty}\left[\frac{2(n-k-1+\alpha)}{(B-A)-\alpha(1-B)}\right]\left|a_{n}\right| \geq 0 .
$$

To show that the function $f$ given by (7) gives the sharp result, we observe that for

$$
\begin{aligned}
z=r e^{\frac{\pi i}{k+2+2 \alpha}} & \frac{(B)}{f_{k}(z)}=1+\frac{(B-A)-\alpha(1-B)}{2(k+\alpha)+(2+A+B)} z^{k+2+2 \alpha} \\
\rightarrow & 1-\frac{(B-A)-\alpha(1-B)}{2(k+\alpha)+(2+A+B)} \\
= & \frac{2(k+\alpha)+(2+A+B)-(B-A)+\alpha(1-B)}{2(k+\alpha)+(2+A+B)} \\
= & \frac{2(1+k+A+\alpha)}{2(k+\alpha)+(2+A+B)}
\end{aligned}
$$

when $r \rightarrow 1^{-}$. Therefore we complete the proof of Theorem 3.1.

Next result can be found in [5].

Corollary 3.1. Let $f$ be given by (1) and satisfies (3) then

$$
\Re\left\{\frac{f(z)}{f_{k}(z)}\right\} \geq \frac{2(1+k+A)}{2 k+2+A+B}, \quad(z \in U) .
$$


The result is sharp for every $k$ with extremal function

$$
f(z)=\frac{1}{z}+\frac{(B-A)}{2 k+2+A+B} z^{k+1}, \quad k \geq 0 .
$$

Proof. Assume that $\alpha=0$.

Moreover, the following result can be found in [8].

Corollary 3.2. Let the assumptions of Theorem 3.1 hold. Then for $f$ of the form (1) satisfies condition

$$
\begin{aligned}
& \sum_{n=1}^{\infty}(n+\mu)\left|a_{n}\right| \leq 1-\mu, \quad(z \in U), \\
& \Re\left\{\frac{f(z)}{f_{k}(z)}\right\} \geq \frac{k+2 \mu}{k+1+\mu}, \quad(z \in U) .
\end{aligned}
$$

The result is sharp for every $k$ with extremal function

$$
f(z)=\frac{1}{z}+\frac{1-\mu}{k+1+\mu} z^{k+1}, \quad k \geq 0
$$

Proof. Assume that $\alpha=0, A=2 \mu-1, B=1$.

Theorem 3.2. Let $f \in \Sigma_{\alpha}$ and

$$
\sum_{n=1}^{\infty}(n+\alpha)[(n+\alpha)(1+B)+(A+1)]\left|a_{n}\right| \leq(1+\alpha)[(B-A)-\alpha(1-B)], \quad(z \in U)
$$

holds, then

$$
\Re\left\{\frac{f(z)}{f_{k}(z)}\right\} \geq \frac{(k+2)(2 k+A+B)+\alpha[(2 k+2+\alpha)(1+B)+2 \alpha(1-B)-B+2 A+1)]}{(k+1)(2 k+2+A+B)+\alpha[(2 k+2+\alpha)(1+B)+(A+1)]} .
$$

The result is sharp for every $k$ with extremal function

$$
f(z)=\frac{1}{z^{1+\alpha}}+\left[\frac{(1+\alpha)[(B-A)-\alpha(1-B)]}{(k+1)(2 k+2+A+B)+\alpha[(2 k+2+\alpha)(1+B)+(A+1)]}\right] z^{k+1+\alpha}, \quad k \geq 0 .
$$


Proof. Let $f \in \Sigma_{\alpha}$. Then we obtain

$$
\begin{aligned}
& \frac{(k+1)(2 k+2+A+B)+\alpha[(2 k+2+\alpha)(1+B)+(A+1)]}{(1+\alpha)[(B-A)-\alpha(1-B)]} \times \\
& {\left[\frac{f(z)}{f_{k}(z)}-\frac{(k+2)(2 k+A+B)+\alpha[(2 k+2+\alpha)(1+B)+2 \alpha(1-B)-B+2 A+1)]}{(k+1)(2 k+2+A+B)+\alpha[(2 k+2+\alpha)(1+B)+(A+1)]}\right.} \\
& =\frac{1+\sum_{n=1}^{k} a_{n} z^{n+2 \alpha+1}+\frac{(k+1)(2 k+2+A+B)+\alpha[(2 k+2+\alpha)(1+B)+(A+1)]}{(1+\alpha)[(B-A)-\alpha(1-B)]} \sum_{n=k+1}^{\infty} a_{n} z^{n+2 \alpha+1}}{1+\sum_{n=1}^{k} a_{n} z^{n+2 \alpha+1}} \\
& :=\frac{1+w_{\alpha}(z)}{1-w_{\alpha}(z)},
\end{aligned}
$$

where

$$
\begin{aligned}
& w_{\alpha}(z)= \\
& \frac{(k+1)(2 k+2+A+B)+\alpha[(2 k+2+\alpha)(1+B)+(A+1)]}{(1+\alpha)[(B-A)-\alpha(1-B)]} \sum_{n=k+1}^{\infty} a_{n} z^{n+2 \alpha+1} \\
& 2+2 \sum_{n=1}^{k} a_{n} z^{n+2 \alpha+1}+\frac{(k+1)(2 k+2+A+B)+\alpha[(2 k+2+\alpha)(1+B)+(A+1)]}{(1+\alpha)[(B-A)-\alpha(1-B)]} \sum_{n=k+1}^{\infty} a_{n} z^{n+2 \alpha+1}
\end{aligned}
$$

and

$$
\left|w_{\alpha}(z)\right| \leq \frac{\frac{(k+1)(2 k+2+A+B)+\alpha[(2 k+2+\alpha)(1+B)+(A+1)]}{(1+\alpha)[(B-A)-\alpha(1-B)]} \sum_{n=k+1}^{\infty}\left|a_{n}\right|}{2-2 \sum_{n=1}^{k}\left|a_{n}\right|-\frac{(k+1)(2 k+2+A+B)+\alpha[(2 k+2+\alpha)(1+B)+(A+1)]}{(1+\alpha)[(B-A)-\alpha(1-B)]} \sum_{n=k+1}^{\infty}\left|a_{n}\right|} .
$$

Since $\left|w_{\alpha}(z)\right| \leq 1$ if and only if

$2\left[\frac{(k+1)(2 k+2+A+B)+\alpha[(2 k+2+\alpha)(1+B)+(A+1)]}{(1+\alpha)[(B-A)-\alpha(1-B)]}\right] \sum_{n=k+1}^{\infty}\left|a_{n}\right| \leq 2-2 \sum_{n=1}^{k}\left|a_{n}\right|$,

this means

$$
\sum_{n=1}^{k}\left|a_{n}\right|+\left[\frac{(k+1)(2 k+2+A+B)+\alpha[(2 k+2+\alpha)(1+B)+(A+1)]}{(1+\alpha)[(B-A)-\alpha(1-B)]}\right] \sum_{n=k+1}^{\infty}\left|a_{n}\right| \leq 1 .
$$

Thus by the assumption of the theorem, the left hand side of (15) is bounded above by

$$
\sum_{n=1}^{\infty}\left[\frac{n(2 n+A+B)+\alpha[(2 n+\alpha)(1+B)+(A+1)]}{(1+\alpha)[(B-A)-\alpha(1-B)]}\right]\left|a_{n}\right|
$$


if

$$
\begin{aligned}
& \frac{1}{(1+\alpha)[(B-A)-\alpha(1-B)]}\left\{\sum_{n=1}^{k}[n(2 n+A+B)+\alpha[(2 n+\alpha)(1+B)+(A+1)]\right. \\
& -(1+\alpha)[(B-A)-\alpha(1-B)]]\left|a_{n}\right|+\sum_{n=k+1}^{\infty}[n(2 n+A+B)+\alpha[(2 n+\alpha)(1+B)] \\
& \left.+(A+1)-(k+1)(2 k+2+A+B)-\alpha[(2 k+2+\alpha)(1+B)+(A+1)]]\left|a_{n}\right|\right\} \geq 0 .
\end{aligned}
$$

Which completes the proof of Theorem 3.2.

The following result can be found in [5].

Corollary 3.3. Let $f$ be given by (1) and satisfies (5) then

$$
\Re\left\{\frac{f(z)}{f_{k}(z)}\right\} \geq \frac{(k+2)(2 k+A+B)}{(k+1)(2 k+2+A+B)}, \quad(z \in U) .
$$

The result is sharp for every $k$ with extremal function

$$
f(z)=\frac{1}{z}+\frac{(B-A)}{(k+1)(2 k+2+A+B)} z^{k+1}, \quad k \geq 0 .
$$

Proof. Assume that $\alpha=0$.

Further, the next result can be found in [8].

Corollary 3.4. Let the assumptions of Theorem 3.2 hold. Then for $f(z)$ of the form (1) satisfies condition

$$
\begin{aligned}
& \sum_{n=1}^{\infty} n(n+\mu)\left|a_{n}\right| \leq 1-\mu, \quad(z \in U), \\
& \Re\left\{\frac{f(z)}{f_{k}(z)}\right\} \geq \frac{(k+2)(k+\mu)}{(k+1)(k+1+\mu)}, \quad(z \in U) .
\end{aligned}
$$

The result is sharp for every $k$ with extremal function

$$
f(z)=\frac{1}{z}+\frac{1-\mu}{(k+1)(k+1+\mu)} z^{k+1}, \quad k \geq 0 .
$$

Proof. Assume that $\alpha=0, A=2 \mu-1, B=1$.

We next determine the bounds for $\Re\left\{\frac{f_{k}(z)}{f(z)}\right\}$ of functions in the classes $\mathcal{S}_{\alpha}(A, B)$ and $\mathcal{C}_{\alpha}(A, B)$. 
Theorem 3.3. Let $f \in \Sigma_{\alpha}$ such that

$$
\sum_{n=1}^{\infty}[(n+\alpha)(1+B)+(A+1)]\left|a_{n}\right| \leq(B-A)-\alpha(1-B), \quad(z \in U)
$$

holds. Then

$$
\Re\left\{\frac{f_{k}(z)}{f(z)}\right\} \geq \frac{2(k+1)+A+B+\alpha(1+B)}{k+2+2 \alpha B}, \quad(z \in U) .
$$

Equalities hold for the functions given by (7).

Proof. Let $f \in \Sigma_{\alpha}$, then we have

$$
\begin{aligned}
\frac{k+2+2 \alpha B}{(B-A)-\alpha(1-B)} & \left\{\frac{f_{k}(z)}{f(z)}-\frac{2(k+1)+A+B+\alpha(1+B)}{k+2+2 \alpha B}\right\} \\
& =\frac{1+\sum_{n=1}^{k} a_{n} z^{n+2 \alpha+1}+\frac{2(k+1)+A+B+\alpha(1+B)}{(B-A)-\alpha(1-B)} \sum_{n=k+1}^{\infty} a_{n} z^{n+2 \alpha+1}}{1+\sum_{n=1}^{k} a_{n} z^{n+2 \alpha+1}} \\
& :=\frac{1+w_{\alpha}(z)}{1-w_{\alpha}(z)},
\end{aligned}
$$

where

$$
w_{\alpha}(z)=\frac{\frac{2(k+1)+A+B+\alpha(1+B)}{(B-A)-\alpha(1-B)} \sum_{n=k+1}^{\infty} a_{n} z^{n+2 \alpha+1}}{2+2 \sum_{n=1}^{k} a_{n} z^{n+2 \alpha+1}+\frac{2(k+1)+A+B+\alpha(1+B)}{(B-A)-\alpha(1-B)} \sum_{n=k+1}^{\infty} a_{n} z^{n+2 \alpha+1}}
$$

with

$$
\left|w_{\alpha}(z)\right| \leq \frac{\frac{2(k+1)+A+B+\alpha(1+B)}{(B-A)-\alpha(1-B)} \sum_{n=k+1}^{\infty}\left|a_{n}\right|}{2-2 \sum_{n=1}^{k}\left|a_{n}\right|-\frac{2(k+1)+A+B+\alpha(1+B)}{(B-A)-\alpha(1-B)} \sum_{n=k+1}^{\infty}\left|a_{n}\right|} .
$$

Note that $\left|w_{\alpha}(z)\right| \leq 1$ if and only if

$$
2\left[\frac{2(k+1)+A+B+\alpha(1+B)}{(B-A)-\alpha(1-B)}\right] \sum_{n=k+1}^{\infty}\left|a_{n}\right| \leq 2-2 \sum_{n=1}^{k}\left|a_{n}\right|
$$

which implies

$$
\sum_{n=1}^{k}\left|a_{n}\right|+\left[\frac{2(k+1)+A+B+\alpha(1+B)}{(B-A)-\alpha(1-B)}\right] \sum_{n=k+1}^{\infty}\left|a_{n}\right| \leq 1 .
$$

From the assumption of the theorem, we can observe that the left hand side of (21) is bounded above by

$$
\sum_{n=1}^{\infty}\left[\frac{2 n+A+B+\alpha(1+B)}{(B-A)-\alpha(1-B)}\right]\left|a_{n}\right| .
$$

Hence the proof.

The following result can be found in [5]. 
Corollary 3.5. Let the assumptions of Corollary 3.1 hold. Then

$$
\Re\left\{\frac{f_{k}(z)}{f(z)}\right\} \geq \frac{2(k+1)+A+B}{k+2}, \quad(z \in U) .
$$

Proof. Assume that $\alpha=0$.

Further, the next result can be found in [8].

Corollary 3.6. Let the assumptions of Corollary 3.2 hold. Then

$$
\Re\left\{\frac{f_{k}(z)}{f(z)}\right\} \geq \frac{k+1+\mu}{k+2}, \quad(z \in U) .
$$

Proof. Assume that $\alpha=0, A=2 \mu-1, B=1$.

In the same manner, we can prove the following result

Theorem 3.4. Let $f$ be given by (1) and satisfies (5) then

$$
\Re\left\{\frac{f_{k}(z)}{f(z)}\right\} \geq \frac{(k+1) 2(2 k+2+A+B)+\alpha \nu}{2(k+1)(k+2)-(B-A)+\alpha \omega}, \quad(z \in U) .
$$

where

$$
\nu:=[(k+1+\alpha)(1+B)+(A+1)+(k+1)(B+1)]
$$

and

$$
\omega:=[(k+1+\alpha)(1+B)+(A+1)+(k+1)(B+1)+(B-A)-(\alpha+1)(1-B)] .
$$

Equalities hold for the function given by (14).

Corollary 3.7. Let the assumptions of Corollary 3.3 hold. Then

$$
\Re\left\{\frac{f_{k}(z)}{f(z)}\right\} \geq \frac{(k+1) 2(2 k+2+A+B)}{2(k+1)(k+2)-(B-A)}, \quad(z \in U) .
$$

Proof. Assume that $\alpha=0$.

Further, the next result can be found in [8].

Corollary 3.8. Let the assumptions of Corollary 3.4 hold. Then

$$
\Re\left\{\frac{f_{k}(z)}{f(z)}\right\} \geq \frac{(k+1)(k+1+\mu)}{(k+1)(k+2)-k(1-\mu)}, \quad(z \in U) .
$$

Proof. Assume that $\alpha=0, A=2 \mu-1, B=1$.

We turn to ratios involving derivatives (see [9]). In the similar manner, we can prove the following results and so the details may be omitted. 
Theorem 3.5. Let $f$ be given by (1) and satisfies (3) with $A=-B$. Then

$$
\begin{gathered}
\Re\left\{\frac{f^{\prime}(z)}{f_{k}^{\prime}(z)}\right\} \geq 0, \quad(z \in U), \\
\Re\left\{\frac{f_{k}^{\prime}(z)}{f^{\prime}(z)}\right\} \geq \frac{1+2 \alpha}{2(1+\alpha)}, \quad(z \in U) .
\end{gathered}
$$

In both cases, the extremal function is given by (7) with $\alpha=0, A=-B$.

Theorem 3.6. Let $f$ be given by (1) and satisfies (5). Then

$$
\begin{aligned}
& \Re\left\{\frac{f^{\prime}(z)}{f_{k}^{\prime}(z)}\right\} \geq \frac{2(k+A+B)+\phi-\alpha[(k+1)(B-A-\alpha(1-B))]}{(2+2 k+A+B)+\phi}, \quad(z \in U), \\
& \Re\left\{\frac{f_{k}^{\prime}(z)}{f^{\prime}(z)}\right\} \geq \frac{2 k+2+A+B+\phi}{2(k+2)+\phi+\alpha[(k+\alpha)(B-A-(\alpha+1)(1-B))-(k+1)(1-B)]}, \quad(z \in U) .(30
\end{aligned}
$$

where

$$
\phi:=\alpha[(k+1+\alpha)(1+B)+(A+1)+(1+B)(k+1)] .
$$

In both cases, the extremal function is given by (14).

Proof. The proof comes immediately from Theorems 3.1 and 3.3 respectively.

Remark 3.1. We note that $\alpha=0$ in Theorems 3.5 and 3.6 coincide with the results obtained in [5].

\section{Acknowledgement}

The work presented here was supported by Fundamental Research Grant Scheme: UKM-ST-01-FRGS0055-2006, Malaysia.

\section{References}

[1] M. L. Mogra, T. R. Reddy, O. P. Juneja, Meromorphic univalent functions, Bull. Austral. Math. Soc. 32 (1985), 161-176.

[2] M. L. Mogra, Hadamard product of certain meromorphic univalent functions, J. Math. Anal. Appl. 157(1991), 10-16.

[3] B. A. Uralegaddi, M. D. Ganigi, Meromorphic convex functions with negative coefficients, J. Math. Res. Exposition 7 (1987), 21-26.

[4] V. Ravichandran, S. Sivaprasad Kumar, M. Darus, On a subordination theorem for a class of meromorphic functions, J. Ineq. Pure Appl. Math., (5)1 Article (8) (2004).

[5] S. Latha, L. Shivarudrappa, Partial sum of meromorphic functions, J. Ineq. Pure Appl. Math. 7, issue 4, article 140, (2006), 1-18. 
[6] R. W. Ibrahim, M. Darus, On subordination theorems for new classes of normalize analytic functions, Appl. Math. Sci. Vol 2, 56, (2008), 2785-2794.

[7] M. Darus, R. W. Ibrahim, Coefficient inequalities for a new class of univalent functions, Lobachevskii J. Math. 29 (2008), 221-229.

[8] H. Silverman, Partial sums of starlike and convex functions, J. Math. Anal. Appl. 209 (1997), 221-227.

[9] T. Rosy, K. G. Subramanian, G. Murugusundaramoorthy, Neighbourhoods and partial sums of starlike functions based on Ruscheweyh derivatives, J. Ineq. Pure Appl. Math. 4, issue 4, article 64, (2003), 1-8.

School of Mathematical Sciences, Faculty of science and Technology, University Kebangsaan Malaysia, Bangi 43600, Selangor Darul Ehsan, Malaysia.

E-mail: rabhaibrahim@yahoo.com

School of Mathematical Sciences, Faculty of science and Technology, University Kebangsaan Malaysia, Bangi 43600, Selangor Darul Ehsan, Malaysia.

E-mail: maslina@ukm.my 